\title{
Characterization of antimicrobial metabolites from medicinal mushrooms against Mango anthracnose pathogen Colletotrichum gloeosporioides (Penz.) Sacc.
}

\author{
M. Gayathiri, G. Thiribhuvanamala*, A.S. Krishnamoorthy, S. Haripriya*, S.B. Akshaya and I. Arumukapravin \\ Department of Plant Pathology, Tamil Nadu Agricultural University, Coimbatore-641003, Tamil Nadu, India \\ *Department of Nanoscience and Technology, Tamil Nadu Agricultural University, Coimbatore-641003, Tamil Nadu, India
}

\section{Article Info}

Article history

Received 13 April 2021

Revised 29 May 2021

Accepted 30 May 2021

Published online 30 June 2021

\section{Keywords}

Mango

Anthracnose

Mycelial inhibition

Antimicrobial activity

Culture filtrate

Ganoderma lucidum

\begin{abstract}
Mango is prone to attack by many diseases and one among them is the anthracnose disease, caused by Colletotrichum gloeosporioides (Penz.) Sacc. which cause both pre and post-harvest yield losses even up to 100 per cent based on prevailing wet humid weather condition. The present investigation has been focused on exploring the possibilities of identifying bioactive compounds from macro basidiomycetes against $C$. gloeosporioides. In the present study, different solvents such as ethyl acetate, diethyl ether, hexane, and chloroform at different concentrations $(0.1$ to 1 per cent) were used to extract secondary metabolites from culture filtrates of medicinal mushrooms, Ganoderma lucidum and Auricularia. polytricha. However, the ethyl acetate extracts of G. lucidum and diethyl ether fraction of $A$. polytricha at 1 per cent concentration showed maximum mycelial growth inhibition of 68.55 per cent and 58.55 per cent, respectively. Characterization of antimicrobial compounds from ethyl acetate extracted culture filtrates of G. lucidum through gas chromatography-mass spectrometry (GC-MS) indicated the presence of novel compound papaverine which recorded the highest peak area, 2.13 per cent with a probability of 90.74 per cent at RT 26.68. Further, the functional groups identified through fourier transform infra-red spectrophotometer (FT-IR) analysis revealed the nature of compounds belonging to aliphatic primary amine, amine salt, thiol, amide, conjugated ketone, alkane, and alcohol. The thin layer chromatography (TLC) studies performed with the ethyl acetate extracted culture filtrate of G. lucidum indicated the appearance of two bands at the $\mathrm{R} f$ value of 0.45 and 0.29 . Further, testing of eluted bands through agar well diffusion assay showed 36.66 and 40.50 per cent inhibition of mycelial growth of C. gloeosporioides, respectively. The standard samples of papaverine (Source: Sigma Aldrich and Compound code: P3510-5g) tested against mycelial growth and conidial germination of C. gloeosporioides exhibited 100 per cent inhibition of mycelial growth of C. gloeosporioides at 1500 ppm and $3000 \mathrm{ppm}$ in agar well diffusion assay and paper disc assay which confirmed the presence of antimicrobial metabolites from culture filtrates of G. lucidum.
\end{abstract}

\section{Introduction}

Mango is susceptible to various diseases, insect pests, and physiological disorders (Sayiprathap et al.,2018). The most destructive diseases are caused by fungi, bacteria, and phytoplasma which results in loss of a quantity of produce (Jha et al.,2010). Among that mango, anthracnose is one of the major devastating calamity due to its pre-harvest as well post-harvest infection and also widely distributed in all mango growing regions of the world. The disease causes a yield loss of 60 per cent, sometimes it extend up to 100 per cent under prevailing wet humid condition (Sharma et al.,2018). Under the current scenario, continuous and judicious application of fungicide against the pathogen has resulted in creating environmental pollution and health problem apart from that, it also leads to the loss of effectiveness of fungicide due to the build-up of fungicide resistance in the pathogen. As an alternative, scientists

Corresponding author: Dr. G. Thiribhuvanamala Associate Professor, Department of Plant Pathology, Tamil Nadu Agricultural University, Coimbatore-641003, Tamil Nadu, India E-mail: ragumala2000@gmail.com Tel.: +91-9629496555

Copyright (c) 2021 Ukaaz Publications. All rights reserved.

Email: ukaaz@yahoo.com; Website: www.ukaazpublications.com have started searching for antimicrobial bioactive compounds from various natural sources to overcome the current situation. In that context, mushrooms are in the limelight and evoked interest globally for their bioactive compounds that find their pharmaceutical and therapeutical values as well as their nutritive value (Poucheret et al., 2006). Mushrooms are the natural blessing in the universe and they possess various natural compounds with bioactive potentials such as antifungal, antibacterial, antiviral, antitumor, antinemic, anti-inflammatory, antiallergic, antiantherogenic, antidiabetic properties (Lindequiste et al.,2005). In this background, the present study was framed with an aim to screen the antimicrobial activity of medicinal mushroom fungi against $C$. gloeosporioides and also involve the characterization of the antimicrobial compounds and identification of their functional groups which help us to develop fungicide formulation.

\section{Materials and Methods}

\subsection{Collection of fungal cultures}

Pure cultures of mango anthracnose pathogen, Collectotrichum gloeosporioides, and medicinal mushroom fungal cultures, Ganoderma lucidum, and Auricularia polytricha were obtained from the Department of Plant Pathology, Tamil Nadu Agricultural University, Coimbatore. 
2.2 Extraction and testing of crude culture filtrates from mycelium of Ganoderma lucidum and Auricularia polytricha

The mycelial disc $(9 \mathrm{~mm})$ from 10 days old culture of A. polytricha and G. lucidum were placed into a $250 \mathrm{ml}$ conical flask containing sterilized potato dextrose (PD) broth. The flasks were kept in an incubator cum shaker at $25^{\circ} \mathrm{C}$ with agitation at $150 \mathrm{rpm}$. After incubation, the mycelial mat was separated from the broth using Whatman filter paper No 1 . Then, the culture filtrate was centrifuged at $10,000 \mathrm{rpm}$ for 10 min. To avoid the bacterial contamination, the supernatant was then filtered through the $0.2 \mu \mathrm{m}$ membrane filter. This extract was used as cell-free crude culture filtrates. The crude culture filtrates were collected from A. polytricha and G. lucidum at various periodical intervals, viz., $10^{\text {th }}, 15^{\text {th }}, 20^{\text {th }}$, and $25^{\text {th }}$ days of inoculation and tested for mycelial inhibition studies.

The extracted crude culture filtrate of G. lucidum and A. polytricha from various periodical intervals $\left(10^{\text {th }}, 15^{\text {th }}, 20^{\text {th }}\right.$ and $25^{\text {th }}$ days $)$ were tested against the pathogen by agar well diffusion technique as described by (Stokes and Ridgway, 1980). The PDA medium was poured into the sterilized plates and allowed to solidify. Later, four wells were made at equal distance leaving $1 \mathrm{~cm}$ space from the edge of the plates containing solidified medium using a sterilized cork borer. A $100 \mu 1$ of crude culture filtrate was pipetted and poured into the wells. Using sterilized cork borer, the $9 \mathrm{~mm}$ mycelial disc of $C$. gloeosporioides from 10 days old culture was placed at the center of the plate and incubated at room temperature $\left(28 \pm 2^{\circ} \mathrm{C}\right)$. Sterile water was served as control instead of using culture filtrate.The results obtained from the mycelial inhibition test from culture filtrates of G. lucidum and A. polytricha at various periodical intervals against the test pathogen were taken for identification of the right stage for a collection of secondary metabolites (Jeeva and Krishnamoorthy, 2018). The percentage inhibition was calculated by using the formula of Vincent, (1947) as explained earlier.

2.3 Testing the solvent extracted secondary metabolites from G. lucidum and A. polytricha against C. gloeosporioides

The efficacy of different solvents used for extraction of secondary metabolites from mushrooms possessing antimicrobial activity varied (Nithya et al., 2013). For this purpose, the 20 days old culture filtrate of $G$. lucidum was extracted with various solvents (ethyl acetate, diethyl ether, chloroform, hexane) and made up to various concentrations of $0.1,0.15,0.2$ per cent to test the desired concentration that could inhibit the maximum mycelial growth of C. gloeosporioides.

2.3.1 Extraction of secondary metabolites from $G$ lucidum and A. polytricha using solvents

Mycelial disc ( $9 \mathrm{~mm}$ ) of G. lucidum and A. polytricha from 10 days old culture plate were taken separately and inoculated into the sterilized PD broth in a $250 \mathrm{ml}$ conical flask. The flasks were kept incubated at $25^{\circ} \mathrm{C}$ with continuous agitation at $120 \mathrm{rpm}$ for 20 days. Based on the previous experiments, the maximum antimicrobial activity was found to be reported at 20 days after inoculation. The extraction of metabolites through solvents was fixed at the $20^{\text {th }}$-day old culture filtrate. After incubation, the mycelial grown culture was separated using Whatman filter paper No 1. The mycelial mat was removed and the aqueous filtrate was collected in a separate conical flask, which is dissolved with an equal volume of solvent and further, the flasks were kept overnight at $150 \mathrm{rpm}$ in a shaker.
The layer of solvent and solution were separated using a separating funnel. Further, the solvent fraction was evaporated using a vacuum flask evaporator. The residue obtained during evaporation was dried and suspended in HPLC grade methanol. The final product of the solution was served as stock to analyze its antimicrobial activity against the pathogen.

2.4 In vitro antimicrobial activity of different solvent extracted antimicrobial metabolites from crude culture filtrates of G. lucidum against the growth of $C$. gloeosporioides

The different solvents (ethyl acetate, diethyl ether, hexane, and chloroform) extracts from crude cell-free mycelial filtrates of G. lucidum were tested separately against mycelial inhibition of C. gloeosporioides by using the agar well diffusion technique (Stokes and Rigdway, 1980). The extracts from various solvents (ethyl acetate, diethyl ether, chloroform, hexane) of G. lucidum was made into various concentration $(0.1 \%, 0.15 \%$, and $0.2 \%)$. The PDA medium was poured into the sterilized plates and allows the plates to solidify. After solidification, four well were made at equal distance leaving $1 \mathrm{~cm}$ space from the periphery of the plate using a sterilized cork borer. $100 \mu 1$ of solvent extracted culture filtrate was pipetted and poured into the well. Using sterilized cork borer, mycelial disc ( $9 \mathrm{~mm}$ ) of $C$. gloeosporioides from 10 days old culture plate was placed at the center of the plate. Sterile water was served as control instead of using solvent extracted culture filtrate. The percentage inhibition was calculated by using the formula (Vincent, 1947).

The particular solvent extracts of crude mycelial extracts of G. lucidum possessing maximum antimicrobial activity against C. gloeosporioides were carried for further studies.

\subsubsection{Testing different concentrations of ethyl acetate solvent extracts of G. lucidum mycelial filtrates on growth of C. gloeosporioides}

Based on the previous experiment, ethyl acetate fraction of secondary metabolite showing the highest mycelial inhibition percentage against the pathogen was selected. To narrow down the effectiveness of ethyl acetate fraction, the concentration, was further increased up to 1 percentage to fix the maximum inhibitory concentration. The antimicrobial activity of different concentrations viz. $0.05 \%, 0.1 \%, 0.2 \%, 0.4 \%, 0.6 \%, 0.8 \%$, and $1 \%$ of ethyl acetate extracts of $G$. lucidum were tested against mycelial growth of $C$. gloeosporioides by agar well diffusion technique with three replications.

2.5 In vitro antimicrobial activity of different solvent extracted antimicrobial metabolites from crude culture filtrates of A. polytricha against $C$. gloeosporioides

The different solvents (ethyl acetate, diethyl ether, hexane, and chloroform) extracts from crude cell-free mycelial filtrates of A. polytricha were tested separately against mycelial inhibition of C. gloeosporioides. The extracts from various solvents (ethyl acetate, diethyl ether, chloroform, hexane) of A. polytricha was made into various concentration $(0.1$ per cent, 0.15 per cent and 0.2 per cent). Further, the different concentrations were tested separately against the mycelial growth of $C$. gloeosporioides by agar well diffusion. The particular solvent extracts of crude mycelial extracts of A. polytricha possessing maximum antimicrobial activity against $C$. gloeosporioides were carried for further studies. 
2.5.1 Testing different concentrations of diethyl ether solvent extracts of $A$. polytricha mycelial filtrates on the growth of C. gloeosporioides

Based on a previous experiment diethyl ether fraction of secondary metabolite of A. polytricha showed the highest mycelial inhibition of $C$. gloeosporioides. To narrow down the effectiveness of the diethyl ether fraction, the concentration was further increased up to 1 percentage to fix the maximum inhibitory concentration.The antagonistic effect of different concentrations of diethyl ether solvent extracts from A. polytricha, viz., $0.05 \%, 0.1 \%, 0.2 \%, 0.4 \%, 0.6 \%$, $0.8 \%$ and $1 \%$ were tested against mycelial growth of $C$. gloeosporioides by agar well diffusion method.

2.6 Identification of antimicrobial compound from mycelial extracts of G. lucidum through GC-MS, FT-IR, and TLC.

From the previous experiments, based on the maximum antifungal activity of ethyl acetate extracts of G. lucidum observed when compared to A. polytricha, an experiment was intended to identify the presence of bioactive molecules from solvent extracted mycelial culture filtrates of G. lucidum against $C$. gloeosporioides. For this purpose, the solvent extracts from mycelial culture filtrates of G. lucidum were subjected to gas chromatography-mass spectrometry (GC-MS) for identification of bioactive compounds. Furthermore, fourier transform - infra-red spectrophotometer (FT-IR) and thin layer chromatography (TLC) analysis were carried out to identify the functional group of such bioactive compounds.

2.6.1 Characterization of antimicrobial compound from ethyl acetate extracted mycelial filtrates of $G$. lucidum through GC-MS analysis

Characterization of biomolecules of cell-free culture filtrate (CFC) condensate and mycelial mat extract of G. lucidum. (Ethyl acetate and methanolic fractions) were done by GC-MS analysis. In this study, the trace GC Ultra and DSQII model MS from Thermo Fisher Scientific Limited were engaged for analysis. The instrument was set as follows: Injector port temperature set to $250^{\circ} \mathrm{C}$, Interface temperature set as $250^{\circ} \mathrm{C}$, source kept at $200^{\circ} \mathrm{C}$. The oven temperature was programmed as available, $70^{\circ} \mathrm{C}$ for $2 \mathrm{~min}, 150^{\circ} \mathrm{C}$ @ $8^{\circ} \mathrm{C} / \mathrm{min}$. up to $260^{\circ} \mathrm{C} @ 10^{\circ} \mathrm{C} / \mathrm{min}$. The split ratio was set as 1:50 and the injector used was in splitless mode. The DB-35 MS non-polar column was used, whose dimensions were $0.29 \mathrm{~mm}$ OD x $0.25 \mu \mathrm{m}$ ID x 30 meters length procured from Agilent Co., USA. Helium was used as the carrier gas at one $\mathrm{Ml} / \mathrm{min}$. The mass spectrum (MS) scan from 50 to $650 \mathrm{Da}$. The source was maintained at $200^{\circ} \mathrm{C}$ and $<40$ motor vacuum pressure. The ionization energy was $70 \mathrm{eV}$. The MS was also having an inbuilt pre-filter, which reduced the neutral particles. NIST4 and WILEY9 are the two inbuilt libraries was found in the data system for searching and matching the spectrum. Those compounds with spectral fit values equal to or greater than 700 were considered identification. Based on the MS data library and comparing the spectrum obtained through.

2.6.2 Identification of nature of antimicrobial compound from ethyl acetate extracted culture filtrates of $G$. lucidum through FT-IR analysis

The chemical nature of the antimicrobial compounds from ethyl acetate extracted mycelial culture filtrate fraction of G. lucidum was identified through FT-IR studies. The ethyl acetate solvent fraction of G. lucidum was prepared as same as mentioned (Jassco FT-IR 6800) and analyses were carried at the Department of Agricultural Microbiology, Tamil Nadu Agricultural University, Coimbatore.

\subsubsection{Identification of antimicrobial from ethyl acetate solvent acetate extracted culture filtrates of $G$. lucidum through TLC studies}

The ethyl acetate extracted solvent fractions of mycelial extracts of G. lucidum were spotted on the silica gel-coated TLC plates at the rate of $20 \mu \mathrm{l} / \mathrm{spot}$.The spots were allowed to dry and the chromatograph was developed using a mobile solvent system, viz Chloroform: Methanol: Distilled water (30:4:1) as described by (Aryantha et al., 2001). The plates were allowed to run until it reaches $3 / 4^{\text {th }}$ position of the plate, then the plates were observed under UV transilluminator at $254 \mathrm{~nm}$. Bands observed in the UV lamp were marked and the $\mathrm{R} f$ value was calculated and recorded.

$$
\mathrm{R} f \text { value }=\frac{\text { Distance traveled by solute }}{\text { Distance travelled by solvent }}
$$

2.6.4 Testing the antifungal activity of TLC eluted compound from ethyl acetate extracted mycelial filtrates of G. lucidum against C. gloeosporioides

The specific bands obtained through TLC were scrapped along with silica coating and suspended in $1 \mathrm{ml}$ HPLC grade methanol. The solution was vortexed for 10-15 min and then further centrifuged at 10,000 rpm for $10 \mathrm{~min}$. The compound associated with methanol were separated and filter through a membrane filter $(0.2 \mu \mathrm{m})$, stored at $4^{\circ} \mathrm{C}$ used for further studies. The antimicrobial activity of the compound was tested against $C$. gloeosporioides by agar well diffusion technique.

2.7 Testing and confirming the antifungal activity of a standard sample of papaverine against $C$. gloeosporioides

From the GC-MS analysis, the compound papaverine from mycelium extracts with a high peak area percentage was identified as an antimicrobial compound. Hence, the standard sample papaverine was purchased from Sigma Aldrich chemicals. The antifungal activity of a standard sample of papaverine was tested against mycelial inhibition and spore germination activity of $C$. gloeosporioides agar well diffusion technique and spore germination assay.

2.7.1 Confirming the antifungal activity of papaverine against mycelial growth of $C$. gloeosporioides by agar well diffusion technique

The standard sample of papaverine was tested against the mycelial growth of $C$. gloeosporioides by agar well diffusion technique. Initially, the papaverine was prepared into different concentrations, viz., 500, 1000, 1500, 2000, and $2500 \mathrm{ppm}$. About $15 \mathrm{ml}$ of PDA medium was poured into a sterilized Petri plate and allowed to solidify. Wells were made at an equal distance leaving $1 \mathrm{~cm}$ from the corner of the Petri plate. A $100 \mu 1$ of different concentrations of papaverine was added @ $100 \mu \mathrm{l}$ per well. Sterile water served as a control. Incubate the plates at room temperature $\left(28 \pm 2^{\circ} \mathrm{C}\right)$ and observations were taken daily and the inhibition percentage of mycelial growth of pathogen was calculated by using the formula derived by Vincent (1947). 


\subsubsection{Testing the effect of papaverine on conidial germination of $C$. gloeosporioides}

The antifungal activity of papaverine was tested against spore germination of C. gloeosporioides by using the cavity slide method. Conidia were collected from 10 days grown-up culture of C. gloeosporioides. The conidial suspension was prepared at a concentration of $1 \times 10^{6}$ conidia $/ \mathrm{ml}$. Based on the preliminary screening results obtained in the previous study, the best concentration of papaverine $(1500 \mathrm{ppm})$ was taken for testing the conidial germination of C. gloeosporioides. A drop of conidia suspension of $C$. gloeosporioides and a drop of a standard sample of papaverine at the desired concentrations (1500 ppm) were added separately into the cavity slide and mixed well. The slides were incubated in the Petri plate containing moist cotton to maintain the humidity for germination of spores. The experiment was replicated four times. The conidial suspension suspended in sterile water served as a control. The conidia were incubated for $6,12,18$, and $24 \mathrm{~h}$ at room temperature $\left(28 \pm 2^{\circ} \mathrm{C}\right)$. Germination of the conidia was observed under a phase-contrast microscope. The per cent inhibition of conidial germination was calculated using the formula (Akhter et al., 2006).

Inhibition of conidial germination (\%)

$$
=\frac{\text { Total no. } \text { of conidia }- \text { No. of conidia germinated }}{\text { Total number of conidia }} \times
$$

\subsection{Statistical Analysis}

All the experiments were performed in triplicate and the treatment mean differences were evaluated with the statistical analyses followed as suggested by (Gomez and Gomez, 1984). Statistical software SPSS was used for the analyses of the data. In the case of zero values, the data were arcsine transformed (1/4n) before statistical analysis.

\section{Results}

\subsection{Testing the crude culture filtrate of mushroom fungi} against mycelial growth of $C$. gloeosporioides

Based on the results, the crude culture filtrate G. lucidum and A. polytricha collected on the $10^{\text {th }}$ day did not exhibit any antifungal activity against the pathogen tested. The crude culture filtrate collected from the $15^{\text {th }}$ day onwards showed inhibition of mycelial growth of $C$. gloeosporioides. However, the culture filtrate collected on the $20^{\text {th }}$ day showed maximum mycelial inhibition of 36.33 per cent and 47.11 per cent of $C$. gloeosporioides with respect to $G$. lucidum and A. polytricha, respectively (Table 1). From the result, it was concluded that the idiophase for the maximum production of antifungal metabolites from G. lucidum and A. polytricha could be the $20^{\text {th }}$ day in the culture filtrate and it was found to be the crucial period for the extraction of metabolites under liquid state fermentation method. Hence, further studies were focused on the extraction of antimicrobial compounds from A. polytricha and $G$. lucidum on the $20^{\text {th }}$ day of the extraction.

\begin{tabular}{|c|c|c|c|c|c|}
\hline \multirow[b]{2}{*}{ S. No. } & \multirow[b]{2}{*}{ Days interval } & \multicolumn{2}{|c|}{ G. lucidum } & \multicolumn{2}{|c|}{ A. polytricha } \\
\hline & & $\begin{array}{l}\text { *Mean mycelial } \\
\text { of the pathogen } \\
(\mathrm{mm})\end{array}$ & $\begin{array}{l}\text { Per cent inhibition } \\
\text { over control }\end{array}$ & $\begin{array}{l}\text { *Mean mycelial } \\
\text { of the pathogen } \\
(\mathrm{mm})\end{array}$ & $\begin{array}{l}\text { Per cent } \\
\text { inhibition over } \\
\text { control }\end{array}$ \\
\hline 1 & $10^{\text {th }}$ day & $90.00(71.61)$ & 0.00 & $90.00(71.61)$ & 0.00 \\
\hline 2 & $15^{\text {th }}$ day & $58.66^{\mathrm{ab}}(49.96)$ & 34.88 & $52.33^{\mathrm{b}}(46.31)$ & 41.88 \\
\hline 3 & $20^{\text {th }}$ day & $57.33^{\mathrm{a}}(49.19)$ & 36.33 & $47.60^{\mathrm{a}}(43.60)$ & 47.11 \\
\hline 4 & $25^{\text {th }}$ day & $59.33^{\mathrm{b}}(50.35)$ & 34.11 & $51.33^{b}(4.74)$ & 43.00 \\
\hline 5 & Control & $90.00(71.61)$ & 0.00 & $90.00(71.61)$ & 0.00 \\
\hline & $\mathrm{SE}(\mathrm{d})$ & 1.265 & - & 1.235 & - \\
\hline & $\mathrm{CD}(p=0.05)$ & 2.854 & - & 2.787 & - \\
\hline
\end{tabular}

Table 1: Efficacy of crude culture filtrates of G. lucidum and A. polytricha against C.gloeosporioides in vitro

Values are the mean of four replications. Means followed by a common letter are not significantly different at $5 \%$ level by DMRT. Values in parenthesis are arcsine transformed values.

3.2 Testing the solvent extracted culture filtrate of $G$. lucidum and $A$. polytricha at different concentration against mycelial growth of $C$. gloeosporioides

In respect of cell-free culture filtrates of G. lucidum, all the concentrations of the solvents tested showed various degrees of mycelial inhibition percentage ranging from 31.11 to 55.55 . Maximum inhibition of mycelial growth ( 41.55 to 55.55 per cent) was observed at 0.2 per cent concentration irrespective of the solvents chloroform, hexane, diethyl ether, and ethyl acetate. However, the ethyl acetate fraction of G. lucidum exhibited a mycelial inhibition percentage of 55.55 at 0.2 per cent concentration followed by diethyl ether, hexane, and chloroform fractions (47.77 per cent, 46.33 per cent, 41.55 per cent, respectively) (Figure 1). From the results, it was obvious that among the solvents used the ethyl acetate fraction of mycelial culture filtrate of G. lucidum showed maximum mycelial inhibition of $C$. gloeosporioides. To narrow down the effectiveness of the ethyl acetate fraction, the concentration was further increased up to 1 per cent to know the maximum inhibitory potential of the antimicrobial compound present in G. lucidum against the mycelium of $C$. gloeosporioides. The results revealed that the inhibition of mycelial growth of $C$. gloeoporioides was observed at all concentrations ranging from 40 per cent to 68.55 per cent inhibition. It is found that the inhibition 
percentage of mycelial growth has been increased from 0.2 to 1 per cent concentration (55.55 to 68.55 per cent). However, the ethyl acetate extracts of $G$. lucidum at 1 per cent concentration showed maximum mycelial growth inhibition of $C$. gloeosporioides $(68.55$ per cent) when compared to control (Figure 2).

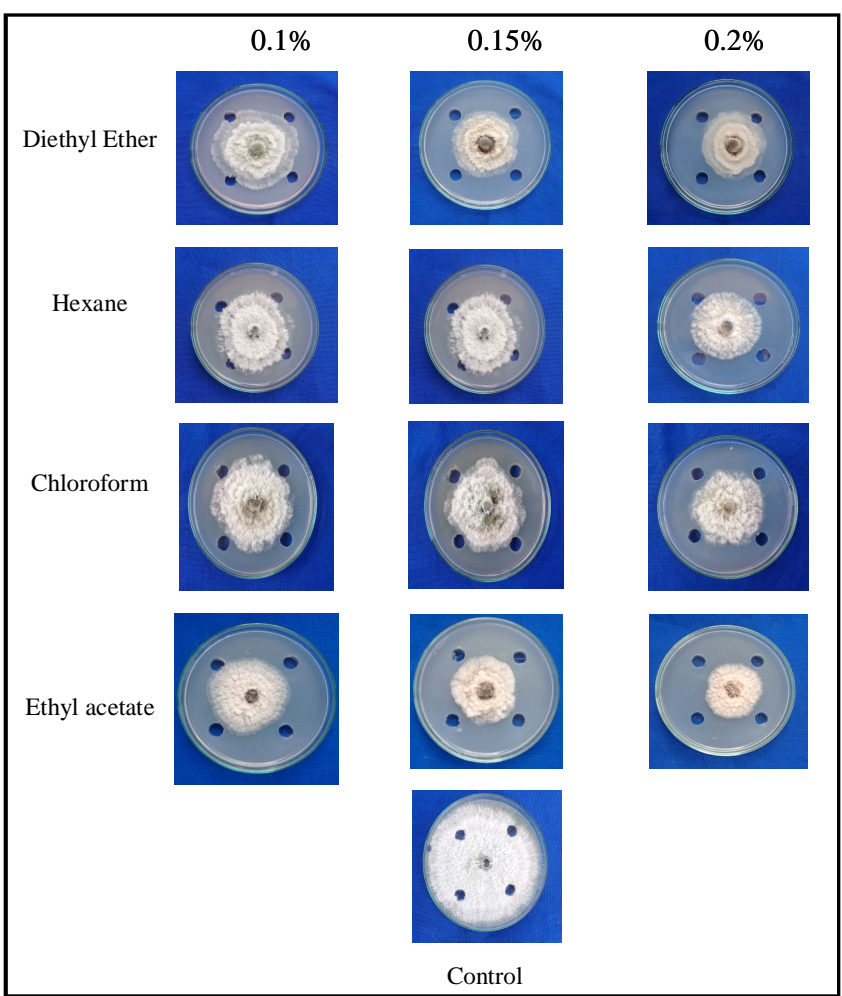

Figure 1: Antimicrobial effect of mycelial culture filtrate of G. lucidum using different solvents against mycelial growth of C. gloeosporioides.

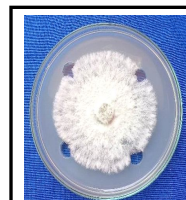

$0.05 \%$

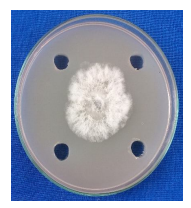

$0.6 \%$

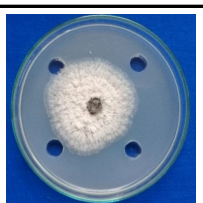

$0.1 \%$

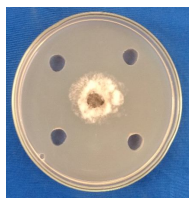

$0.8 \%$

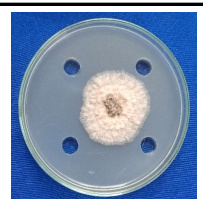

$0.2 \%$

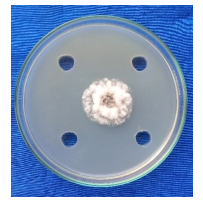

$1 \%$

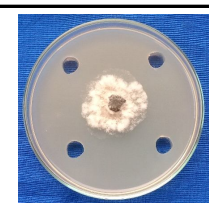

$0.4 \%$

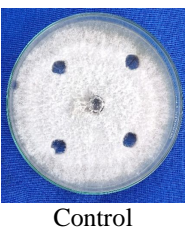

Figure 2: Testing the Ethyl acetate extracted crude mycelial extracts of G. lucidum against mycelial growth of Colletotrichum gloeosporioides.

Similarly, maximum inhibition of mycelial growth (35.22 to 46.33 per cent) was observed at 0.2 per cent concentration irrespective of the solvents diethyl ether, hexane, chloroform, and ethyl acetate. However, the diethyl ether extracts of A. polytricha showed maximum inhibition percentage (46.33 per cent) of mycelial growth of C. gloeosporioides followed by hexane (41.11 per cent), ethyl acetate fraction (38.88 per cent), and chloroform (35.22 per cent) at 0.2 per cent concentration (Table 2). Hence, to find out the effective concentration and maximum inhibitory potential, the concentration of diethyl ether extracts of A. polytricha was further increased up to 1 per cent. The result showed that the mycelial growth inhibition of $C$. gloeoporioides was observed at all concentrations ranging from 35.22 per cent to 58.55 per cent inhibition. However, the inhibition percentage was directly proportional to the higher concentration that exhibited maximum mycelial inhibition of A. polytricha to 58.55 percentage at 1 per cent concentration (Figure 3).

The result revealed that the ethyl acetate extracted secondary metabolites from mycelial extracts of G. lucidum showed maximum mycelial inhibitory potential than A. polytricha at 1 per cent concentration. Hence, G. lucidum was subjected to further studies.

Table 2: Testing crude mycelial culture filtrates of A. polytricha against mycelial growth of C. gloeosporioides

\begin{tabular}{|c|c|c|c|c|c|c|c|c|}
\hline \multirow{2}{*}{$\begin{array}{l}\text { Solvents } \\
\text { Concentration } \\
(\%)\end{array}$} & \multicolumn{2}{|c|}{ Diethyl ether } & \multicolumn{2}{|c|}{ Ethyl acetate } & \multicolumn{2}{|c|}{ Chloroform } & \multicolumn{2}{|c|}{ Hexane } \\
\hline & $\begin{array}{l}\text { Mycelial } \\
\text { growth } \\
(\mathrm{mm})\end{array}$ & PI & $\begin{array}{l}\text { Mycelial } \\
\text { growth } \\
(\mathrm{mm})\end{array}$ & PI & $\begin{array}{l}\text { Mycelial } \\
\text { growth } \\
(\mathrm{mm})\end{array}$ & PI & $\begin{array}{l}\text { Mycelial } \\
\text { growth } \\
(\mathrm{mm})\end{array}$ & PI \\
\hline 0.1 & $\begin{array}{l}54.8^{b c} \\
(47.73)\end{array}$ & 38.00 & $\begin{array}{l}61.5^{\mathrm{b}} \\
(51.63)\end{array}$ & 31.66 & $\begin{array}{l}68.5^{c} \\
(55.86)\end{array}$ & 23.88 & $\begin{array}{l}60.3^{c} \\
(50.92)\end{array}$ & 33.00 \\
\hline 0.15 & $\begin{array}{l}53.3^{\mathrm{b}} \\
(46.87)\end{array}$ & 40.77 & $\begin{array}{l}56.6^{a} \\
(48.77)\end{array}$ & 37.77 & $\begin{array}{l}61.1^{\mathrm{b}} \\
(51.41)\end{array}$ & 32.11 & $\begin{array}{l}56.3^{b} \\
(48.60)\end{array}$ & 37.44 \\
\hline 0.2 & $\begin{array}{l}48.3^{a} \\
(44.00)\end{array}$ & 46.33 & $\begin{array}{l}55.0^{\mathrm{a}} \\
(47.85)\end{array}$ & 38.88 & $\begin{array}{l}58.3^{a} \\
(59.48)\end{array}$ & 35.22 & $\begin{array}{l}53.0^{a} \\
(46.70)\end{array}$ & 41.11 \\
\hline Control & $\begin{array}{l}90.0^{\mathrm{d}} \\
(71.61)\end{array}$ & 0.00 & $\begin{array}{l}90.0^{c} \\
(71.61)\end{array}$ & 0.00 & $\begin{array}{l}90.0^{d} \\
(71.61)\end{array}$ & 0.00 & $\begin{array}{l}90.0^{d} \\
(71.61)\end{array}$ & 0.00 \\
\hline $\mathrm{SE}(\mathrm{d})$ & 0.708 & - & 0.724 & - & 0.732 & - & 0.708 & - \\
\hline $\mathrm{CD}(p=0.05)$ & 1.876 & - & 1.807 & - & 2.030 & - & 1.767 & - \\
\hline
\end{tabular}

Values are the mean of four replications. Means followed by a common letter are not significantly different at 5\% level by DMRT. Values in parenthesis are arcsine transformed values 


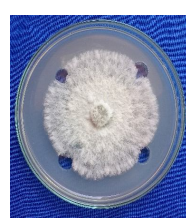

$0.05 \%$

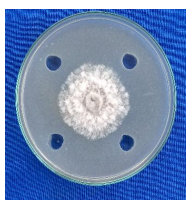

$0.6 \%$

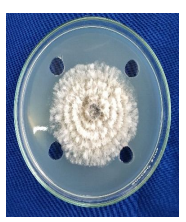

$0.1 \%$

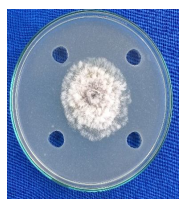

$0.8 \%$

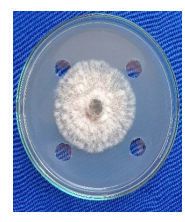

$0.2 \%$

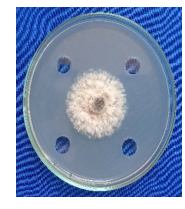

$1 \%$

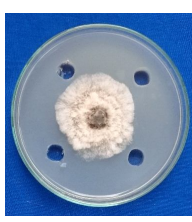

$0.4 \%$

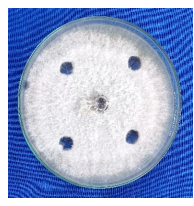

Control
Figure 3: Testing diethyl ether extracted crude mycelial extract of A. polytricha against mycelial growth of C. gloeosporioides.

3.3 Identification and characterization of an antifungal compound from ethyl acetate extracts of mycelial filtrates of G. lucidum through GC-MS analysis

Characterization of biomolecules of cell-free culture filtrate condensate of G .lucidum (ethyl acetate fractions). GC-MS analysis revealed the presence of various compound such as Formic acid, 2propenyl ester, 1, 4, 7, 10, 13, 16, 19 - Heptaoxa-2cycloheneicosanone, Pentaethylene glycol,n-Hexadecanoic acid, Heptaethylene glycol, 9-Decanoic acid, 2,-dimethyl through GCMS analysis. Among the compound, Papaverine is reported to have antiviral activity, n-Hexadecanoic acid with antibacterial and antifungal activity, Pentaethylene glycol, Heptaethylene glycol, and Formic acid, 2-propenyl ester, 1,4,7,10,13,16, 19- Heptaoxa2-cycloheneicosanone with antifungal activity, and 9-Decenoic acid with antinemic property. Among several compounds characterized, papaverine has recorded the highest peak area of about 2.13 per cent with the probability of 90.74 per cent at RT 26.68 was used for further studies owing to its antimicrobial activity (Table 3 ).

3.4 Determination of functional groups of antimicrobial compounds of ethyl acetate fractions of G. lucidum mycelial culture filtrates through FT-IR analysis

FT-IR analysis of ethyl acetate solvent fraction of G. lucidum mycelial culture filtrates exhibited the distribution of functional group within the organic fractions. The spectrum indicated that the leading bands were observed in the regions between 632.537 and $3309.25 \mathrm{~cm}^{-1}$. The peak in the spectrum reveals the presence of aliphatic primary amine, amine salt, thiol, amide, conjugated ketone, alkane, alcohol. The presence of different functional groups indicated the existence of a variety of potential biomolecules in mycelial culture filtrates of G. lucidum (Figure 4).

\subsection{Identifying the bioactive molecules from ethyl acetate solvent extracts of mycelial culture filtrates of $G$ lucidum through TLC studies}

The TLC analysis carried out for the ethyl acetate solvent extracts of $G$. lucidum mycelial culture filtrates using the mobile phase Chloroform: Methanol: Water at the proportion 30:4:1 indicated the appearance of two bright bands under a UV lamp at the $R_{f}$ value of 0.45 and 0.39 (Figure 5a). Further, the bands (band 1 and band 2) were eluted and their antimicrobial activity was tested against $C$. gloeosporioides using the agar well diffusion technique. The results indicated that bands 1 and 2 ( $R_{f}$ value- 0.45 and 0.29 respectively) eluted from the TLC plate showed inhibition of mycelial growth of $C$. gloeosporioides in the range of 36.66 per cent and 40.50 per cent, respectively ( Figure $5 \mathrm{~b}$ ). Based on the result, it is obvious that band 2 possessed maximum antifungal activity against $C$. gloeosporioides when compared with band 1.

Table 3: Characterization of antimicrobial compounds from ethyl acetate solvent extracts from the mycelial culture filtrate of G. lucidum

\begin{tabular}{|c|c|l|c|c|l|}
\hline S.No. & RT & Compound weight $(\mathbf{g} / \mathbf{m l})$ & Molecular formula & Molecular & Biological activity \\
\hline 1. & 4.07 & Formic acid,2-propenyl ester & 46.025 & $\mathrm{CH}_{2} \mathrm{O}_{2}$ & $\begin{array}{l}\text { Antifungal activity against } \\
\text { Candida albicans and } \\
\text { C. guillermondii }\end{array}$ \\
\hline 2. & 15.61 & $\begin{array}{l}1,4,7,10,13,16,19 \text { Heptaoxa-2- } \\
\text { cycloheneicosanone }\end{array}$ & 322.35 & $\mathrm{C}_{14} \mathrm{H}_{26} \mathrm{O}_{8}$ & - \\
\hline 3. & 16.86 & Pentaethylene glycol & 238.28 & $\mathrm{C}_{10} \mathrm{H}_{22} \mathrm{O}_{6}$ & $\begin{array}{l}\text { Antifungal activity against } \\
\text { C. graminicola and } \text { Fusarium spp }\end{array}$ \\
\hline 4. & 26.68 & Papaverine & 375.80 & $\mathrm{C}_{20} \mathrm{H}_{21} \mathrm{NO}_{4}$ & $\begin{array}{l}\text { Antifungal activity and } \\
\text { antimicrobial activity }\end{array}$ \\
\hline 5. & 20.68 & n-Hexadecanoic acid & 256.43 & $\mathrm{C}_{16} \mathrm{H}_{32} \mathrm{O}_{2}$ & Antifungal, antibacterial \\
\hline 6. & 25.47 & Heptaethylene glycol & 326.38 & $\mathrm{C}_{14} \mathrm{H}_{30} \mathrm{O}_{8}$ & $\begin{array}{l}\text { Antifungal activity against } \\
\text { C. graminicola and Fusarium } \text { spp. }\end{array}$ \\
\hline
\end{tabular}

3.6 Confirming the antifungal activity of papaverine (Standard sample) against mycelial growth of $C$. gloeosporioides by agar well diffusion technique

From the GC-MS analysis, the bioactive compound papaverine from mycelial extracts of G. lucidum was identified with a high peak area percentage. Hence, the standard sample of papaverine was purchased from Sigma Aldrich chemicals (Compound code: P3510$5 \mathrm{~g}$ ). tested against mycelial inhibition and spore germination activity of C. gloeosporioides indicated that all the concentrations of papaverine showed inhibition of mycelial growth of $C$. gloeosporioides. Interestingly, the compound exhibits a higher degree of inhibition even at a lower concentration. However, 1500 ppm 
was the minimum concentration that was sufficient enough to completely inhibit the mycelial growth of $C$. gloeosporioides. Moreover, the mycelial pattern of the pathogen in treatment was observed. The morphological changes of cultural characters were observed as breakage of the mycelial disc, formation of thin mycelial pattern with inhibition zone, complete growth arrestment whereas control showed normal growth of mycelium in concentric patterns (Figure 6).

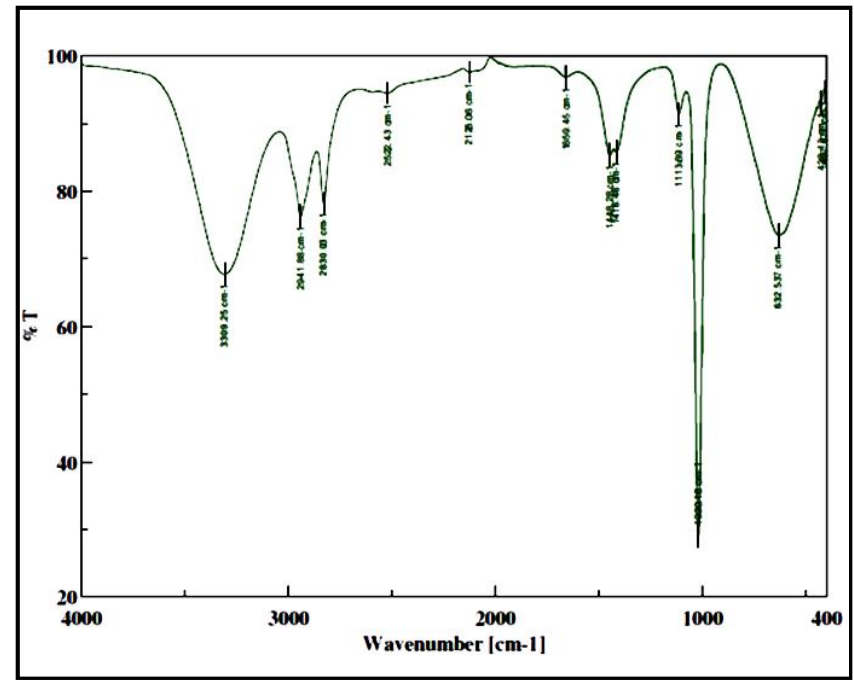

Figure 4: Identification of functional groups of compounds from Ethyl acetate solvent fractions of $G$. lucidum mycelial culture filtrates.

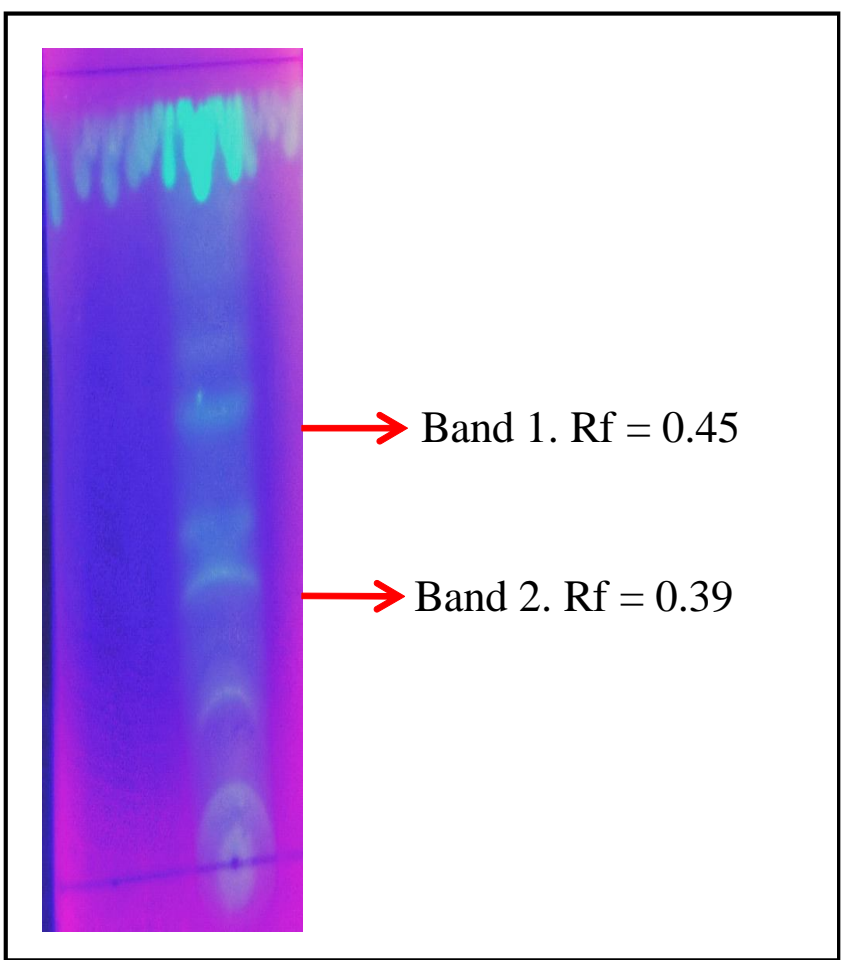

Plate 5a: Detection of antimicrobial compounds from ethyl acetate extracts of $G$. lucidum culture filtrates through TLC.

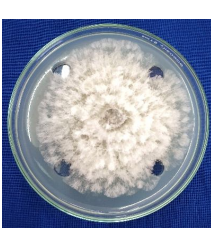

Band 1

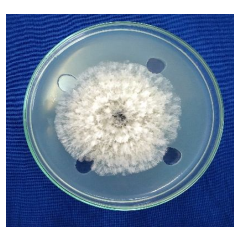

Band 2

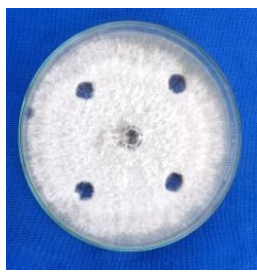

Control

Plate 5b:Antimicrobial activity of compound eluted from TLC

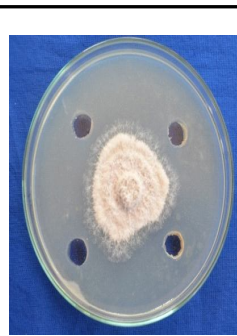

$500 \mathrm{ppm}$

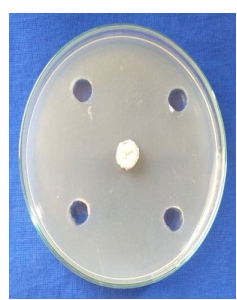

$2000 \mathrm{ppm}$

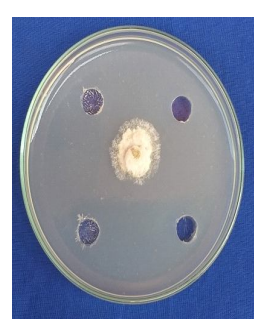

$1000 \mathrm{ppm}$

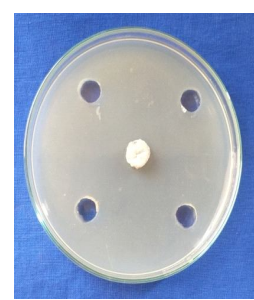

$2500 \mathrm{ppm}$

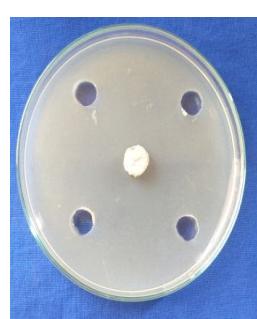

$1500 \mathrm{ppm}$

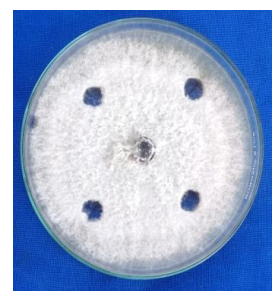

Control
Figure 6: Testing the antifungal efficacy of papaverine at different concentrations against mycelial growth of C. gloeosporioides by agar well diffusion assay.

3.6.1 Testing the effect of papaverine (Standard sample) at an optimum concentration $(1500$ ppm $)$ on conidial germination of $C$. gloeosporioides

The antifungal activity of papaverine tested against $C$. gloeosporioides by cavity slide method revealed that the papaverine at $1500 \mathrm{ppm}$ effectively reduced the conidial germination of C. gloeosporioides after $6 \mathrm{~h}$ of incubation, while the untreated conidia showed reasonable germination at this time. After $12 \mathrm{~h}$ of incubation in papaverine, the germination of conidia was only 13.77 numbers with a 51.34 per cent reduction in conidial germination per cent whereas that of control conidia was 26.82 in number. After $24 \mathrm{~h}$, the germination of spores incubated in papaverine was only 26.74 with 53.11 germination per cent while germination of the untreated conidia of $C$. gloeosporioides was higher in number $(50.34)$. Therefore, it was concluded that the standard sample of papaverine possesses both antifungal as well as inhibition of spore germination (Table 4). 
Table 4: Testing the effect of papaverine (standard) on germination of spores of C. gloeosporioides

\begin{tabular}{|l|ll|l|}
\hline \multirow{2}{*}{ Time interval } & \multicolumn{2}{|c|}{ Conidial } & $\begin{array}{l}\text { Reduction } \\
\text { in germination } \\
\end{array}$ \\
\cline { 2 - 3 } & germination of C. gloeosporioides at $\mathbf{1 5 0 0} \mathbf{~ p p m}$ & Over Control (\%) \\
\hline $6 \mathrm{~h}$ & $0.00(0.57)$ & $0.00^{\mathrm{c}}(0.57)$ & 0.00 \\
\hline $12 \mathrm{~h}$ & $26.82(31.19)$ & $13.77^{\mathrm{b}}(21.77)$ & 51.34 \\
\hline $24 \mathrm{~h}$ & $50.34(45.19)$ & $26.74^{\mathrm{a}}(31.12)$ & 53.11 \\
\hline $\mathrm{SE}(\mathrm{d})$ & - & 0.317 & - \\
\hline $\mathrm{CD}(p=0.05)$ & - & 0.903 & - \\
\hline
\end{tabular}

Values are the mean of four replications.

Means followed by a common letter are not significantly different at 5\% level by DMRT.

Values in parenthesis are arcsine transformed values.

\section{Discussion}

Fungal secondary metabolites are playing major role in inhibiting growth of food borne and clinical pathogens. An understanding on the antifungal metabolites of macrobasiomycetes against plant pathogens is the need of current situation owing to the harmful effects of fungicides. In the present investigation, testing of crude culture filtrates from G. lucidum and A. polytricha at various periodical intervals $10^{\text {th }}, 15^{\text {th }}, 20^{\text {th }}$ and $25^{\text {th }}$ days against $C$. gloeosporioides indicated that the crude culture filtrate collected on $10^{\text {th }}$ day did not exhibit any antifungal effect against the target pathogen. The culture filtrate extracted from the $20^{\text {th }}$ day showed maximum mycelial inhibition of $36.33 \%$ and $47.11 \%$ of C. gloeosporioides with respect to G. lucidum and A. polytricha, respectively which show that maximal secretion of antifungal metabolites takes place on the $20^{\text {th }}$ day. In a similar study, Imtiaj and Lee (2007) analyzed the cell-free culture filtrates of Cordyceps sobolifera, Pycnoporus cinnabarinus, P. coccineus, Oudemansiella mucida, and Sterium ostrea collected on the $20^{\text {th }}$ day against Botrytis cinerea, C. gloeosporioides, and C. miyabeanus. The result depicted that the culture filtrate of $O . m u c i d a$ showed higher mycelial inhibition of 63.01 per cent in C. gloeosporioides while in B. cinerea, the culture filtrate of $C$. sobolifera had exhibited a higher mycelial inhibition percentage of 43.48 and in the case of $C$. miyabeanus, the culture filtrate of $O$. mucida showed higher inhibition percentage of $63.01 \%$, followed by $P$. coccineus $(59.90 \%)$. Priya et al. (2019) reported the antimicrobial activity of crude culture filtrate collected on the $20^{\text {th }}$ day from L. edodes, G. lucidum, C. sinensis, and A. polytricha against $C$. capsici when tested by agar well diffusion assay. Jeeva and Krishnamoorthy (2018) reported that the cell-free culture filtrate (CFC) of Coprinopsis sinensis collected on the $20^{\text {th }}$ day after inoculation showed maximum mycelial inhibition of Fusarium brachygibbosum (28.11 per cent), F. o. f. sp. cubense (39.33), and F. o. f. sp. lycopersici (31.00 per cent) as compared to other days. Similarly, the cell-free culture filtrates of C. comatus, L. edodes, Tremella aurantialba, and Clitocybe spp. collected on the $20^{\text {th }}$ day had exhibited good antifungal effects against Phytophthora capsici (Chen and Huang, 2010). It is obvious to note the result which indicate that the mushroom fungi G. lucidum and A. polytricha secreted minimum quantities of bioactive compound in the culture filtrate since the inhibition percentage was only up to $47.11 \%$. On the other hand, this led us to take up a study on the extraction of bioactive antifungal metabolites from the culture filtrate by using various polar and non-polar solvents. Nithya et al. (2013) mentioned that the efficacy of antimicrobial activity of secondary metabolites was varied based on the polarity of the solvent. Moreover, the selection of solvent is a crucial factor for the extraction of metabolite as it is based on the chemical nature and dissolving capacity of a specific compound. Based on the polarity, different solvents such as ethyl acetate, diethyl ether, hexane, and chloroform were selected and the effectiveness against the target pathogen was observed by the mycelial inhibition test.The antimicrobial screening of mycelial extracts of G. lucidum and A. polytricha showed that the inhibition percentage was directly proportional to the higher concentration. However, the ethyl acetate and diethyl ether fraction of G. lucidum and A. polytricha exhibited maximum inhibition of 68.55 per cent and 58.55 per cent at an increased concentration of 1 per cent which would have extracted more of phenols, terpenoids, enzymes, and proteins. Ethyl acetate performs well in the secondary metabolite extraction because it contains two important chemical and biological characteristics together with medium polarity and minimum toxicity which can help to extract many biologically active compounds (polar and non-polar) as reported by Kumar et al. (2013). Moreover, Ganoderma spp. found to perform differently based on the solvent used for secondary metabolite extraction. This is evidenced by the findings of (Jonathan and Fasidi, 2003) where they reported that the methanol and ethanol extraction of metabolite performed well against the target pathogen than the water extract. On that basis Fujita et al.(2005) reported that the ethanol-based solvent performed better in extracting bioactive compounds and also (Cowan, 1999) stated that more active compounds not soluble in water but highly soluble in low polarity compound which yields effective extraction of bioactive compound. Shahid et al. (2016) found that the acetone extract fraction of $G$. lucidum exhibited maximum antifungal activity than a methanolic fraction as evidenced by inhibition against Fusarium oxysporum (64\%) and 57\% inhibition against Alternaria alternata at 20\% concentration. Stojkoviæ et al. (2013) indicated that the ethanolic extract of $C$. comatus showed antifungal activity against Aspergillus fumigatus, A.ochraceus, A.versicolor Trichoderma viride, Penicillium funiculosum, $P$. ochrochloron, and $P$. verrucosum var. cyclopium. Similar to our results, Radhajeyalakshmi et al,(2010) extracted of antimicrobial compound from A. polytricha, L. edodes and $V$. volvacea using chloroform, ethyl acetate, ether and methanol against plant pathogenic fungus such as Alterneria solani, Rhizoctonia solani, C. capsici and Pythium aphanidermatum and the reported that ethyl acetate was the best solvent with a maximum inhibition of $45 \mathrm{~mm}$ against the targeted pathogen. 
In this study, the ethyl acetate solvent extracts from mycelial culture filtrates of G. lucidum subjected to GC-MS for identification of bioactive compounds revealed that the presence of various compounds, and among them papaverine recorded the highest peak area of about $2.13 \%$ with a probability of 90.74 per cent at RT 26.68. Furthermore, papaverine is known to possess antiviral activity and is used in human medicine (www.ncbi.com). The peak in the spectrum of the FT-IR analysis revealed the presence of different functional groups, viz., aliphatic primary amine, amine salt, thiol, amide, conjugated ketone, alkane, alcohol which is an indication for the existence of a variety of potential biomolecules in mycelial culture filtrates of G. lucidum. Similar to this study, Elumba et al. (2013) carried out the FT-IR analysis of the solvent fraction of $G$. lucidum revealed the presence of various functional groups include primary amine, amine salt, thiol, ketone, alkane, and alcoholic group. Further, in our study, the TLC analysis for the ethyl acetate solvent extracts of mycelium of $\mathrm{G}$. lucidum indicated the appearance of two bright bands with $\mathrm{R}_{\mathrm{f}}$ value 0.45 and 0.29 and both the band 1 and 2 eluted from the TLC plate showed the inhibition of mycelial growth of $C$. gloeosporioides in the range of 36.66 per cent and 40.50 per cent, respectively. Similarly, Priya (2019) carried the TLC analysis for the chloroform fraction of G. lucidum revealed the presence of a band with $\mathrm{R} f$ value 0.27 that had antifungal activity against C.capsici, fruit rot pathogen of chilli.

The compound papaverine is a benzylisoquinoline alkaloid that is reported to have strong antimicrobial activity and also act against viral pathogens in humans (Aiyegoro and Okoh, 2009), but it is so far not exploited against fungal pathogens. The standard sample of papaverine tested against $C$. gloeosporioides by agar well diffusion technique at different concentrations (500 to $3500 \mathrm{ppm}$ ) indicated that the $1500 \mathrm{ppm}$ concentration was the minimum concentration required for the complete inhibition of mycelial growth of $C$. gloeosporioides. Papaverine induced different monocultural changes including breakage of the mycelial disc, dense mycelial growth at the center of the plate, mycelial malformation, formation of the thin mycelial pattern, distortion, and restricted growth of mycelium and. also, the conidial germination inhibition was tested at different hours intervals (from $6 \mathrm{~h}$ to $24 \mathrm{~h}$ interval) by cavity slide method and the result showed that although the conidia germinated at $24 \mathrm{~h}$ of incubation the germination percentage was significantly reduced to 53.11 per cent when compared to control. Probably, this is the first report of papaverine exhibiting antifungal activity against C. gloesporioides.

\section{Conclusion}

Macrobasidiomycetes contain ample bioactive compounds which needs to be properly tapped and identified for the management of plant diseases. The export of mangoes are greatly affected due to anthracnose disease that deteriorates the fruit qulaity. Also, fungicide treated mangoes are not preferred in the international market so an alternate ecofriendly strategy identification of green chemicals will be the only solution in future. In this context, from the present study, it is well proven that for G. lucidum secretes secondary metabo-lites that possess antifungal compound papaverine that could be used further to develop fungicidal formulations for the management of C. gloeosporioides of Mango.

\section{Acknowledgements}

The authors thank the university and ICAR-All India Coordinated Research Project on Mushroom for providing the research facilities to carry out this work.

\section{Conflict of interest}

The authors declare that there is no conflicts of interest relevant to this article.

\section{References}

Aiyegoro, O.A. and Okoh, A.I. (2009). "Use of bioactive plant products in combination with standard antibiotics: Implications in antimicrobial chemotherapy". J. Med. Plants Res., 3(13):1147-1152.

Akhter, N.; Begum, M.F.; Alam, S. and Shah Alam, M.D. (2006). "Inhibitory effect of different plant extracts, cow dung and cow urine on conidial germination of Bipolaris sorokiniana". J. Bio. Sci., 14:87-92.

Ansari, M.N. (2020). Assessment of antidiarrheal, antispasmodic and antimicrobial activities of methanolic seeds extract of Peganum harmala L. (Nitrariaceae). J. Pharma. Res. Int., pp:74-82.

Chen, J.T. and Huang, J.W. (2010). "Antimicrobial activity of edible mushroom culture filtrates on plant pathogens". Plant Pathol Bull., 4:261-270

Cowan, M.M. (1999). "Plant products as antimicrobial agents". Clinic Microbiol Rev., 12(4):564-582.

Elumba, Z.S.; Franco G.T. and Roberto, M.M. (2013). "DNA-binding activity and in vivo cytotoxicity of Ganoderma applanatum (Pers.) Pat. supercritical-CO2 extracts". Afric. J. Microbiol. Res., 7(3):202-210.

Fujita, R.J.; Liu, K.S.; Fumiko, K.; Kiyoshi, N.; Shoichiro, K.; Chie, U.; Hisatoshi, T.; Shuhei, K. and Yoshitaro, S. (2005). "Anti-androgenic activities of Ganoderma lucidum”. J. Ethnopharm., 102(1):107-112.

Gomez, K.A. and Gomez, A.A. (1984). Statistical procedures for agricultural research: John Wiley and Sons., New York, pp:690.

Imtiaj, A. and Lee, T.S. (2007). "Screening of antibacterial and antifungal activities from Korean wild mushrooms". World J. Agri. Sci., 3(3):316-321.

Jeeva, S. and Krishnamoorthy, A.S. (2018). "Antifungal Potential of Mycomolecules of Coprinopsis cinerea (Schaeff) S. Gray s. lat. against Fusarium spp." Mad. Agri. J., 105:1-3.

Jha, S.N.; Narsaiah, K.; Sharma,A.D.; Singh, M.; Bansal, S. and Kumar. R. (2010). "Quality parameters of mango and potential of non-destructive techniques for their measurement: A review." J. Food Sci. Technol., 47(1): $1-14$.

Jonathan, S.G. and Awotona, F. (2010). "Studies on antimicrobial potentials of three Ganoderma species." Afric. J. Biomed. Res., 13(2):131139.

Kumar, S.; Nutan, K. and Proksch, P. (2013). "Identification of antifungal principle in the solvent extract of an endophytic fungus Chaetomium globosum from Withania somnifera." Springer Plus., 2(1):37.

Lindequist, U.; Niedermeyer, T. H. and Wolf-Dieter Jülich. (2005). “The pharmacological potential of mushrooms." Evid. Complement. Alternat. Med., 2(3):289-299.

Nithya, M.; Ambikapathy, V. and Panneerselvam, A. (2013). "Studies on antimicrobial potential of different strains of Ganoderma lucidum (Curt.: Fr.) P”. Int. J. Pharma. Sci. Rev. Res., 21(2):56.

Priya, K. (2019). "Characterization of antimicrobial compounds from mushroom fungi and medicinal plants against Colletotrichum capsici (syd.) Butler and Bisby, an inciting agent of chilli anthracnose." M.sc (Ag)Thesis Tamil Nadu Agric Univ., Coimbatore, India, pp:3646. 
Priya, K.; Thiribhuvanamala, G.; Kamalakannan, A. and Krishnamoorthy, A.S (2019). "Antimicrobial activity of biomolecules from mushroom fungi against colletotrichum capsici (Syd.) Butler and Bisby, the Fruit Rot. Pathogen. of Chilli." Int. J. Cur. Microbiol. Appl. Sci., 8(6):1172-1186.

Radhajeyalakshmi, R.; Velazhahan, R. and Prakasam, V. (2012). "In vitro evaluation of solvent extracted compounds from edible macromycetes against phytopathogenic fungi." Arch. Phytopathol. Plant Protec., 45(3):293-300.

Shahid, A.A.; Muhammad, A; Muhammad, S. and Muhammad, A. (2016). "Antifungal potential of Ganoderma lucidum extract against plant pathogenic fungi of Calendula officinalis L". Int. Conf. Biol. Chem. Env. Sci., (BCES-2016) March.
Sharma, G.; Gryzenhout, M.; Hyde, K.D.; Pinnaka, A.K. and Shenoy. B.D. (2015). "First report of Colletotrichum asianum causing mango anthracnose in South Africa." Plant Disease, 99(5):725-725.

Stojkovic, D.; Filipa S. R.; Lillian, B; Jasmina, G.A.C.; Griensven, L.J.V.; Marina, S. and Isabel C.F. (2013). "Nutrients and non-nutrients composition and bioactivity of wild and cultivated Coprinus comatus (OF Müll.) Pers.” Food Chem. Toxicol., 59:289-296.

Stokes, E.J. and Ridgway, G.L. (1980). “Antibacterial drugs.” Clinical Bacteriology, pp:200-254.

Vincent, J.M. (1947). "Distortion of fungal hyphae in the presence of certain inhibitors." Nature, 159(4051):850-850.

M. Gayathiri, G. Thiribhuvanamala, A.S. Krishnamoorthy, S. Haripriya, S.B. Akshaya and I. Arumukapravin. (2021). Characterization of antimicrobial metabolites from medicinal mushrooms against Mango anthracnose pathogen Colletotrichum gloeosporioides (Penz.) Sacc.. Ann. Phytomed., 10(1):185-194. http://dx.doi.org/10.21276/ ap.2021.10.1.19 